\title{
A molecular dynamics simulation scenario for studying solvent-mediated interactions of polymers and application to thermoresponse of $\operatorname{poly}(N$-isopropylacrylamide) in water
}

\author{
Edder J. García, Debdip Bhandary, Martin T. Horsch, and Hans Hasse*
}

Laboratory of Engineering Thermodynamics (LTD), University of Kaiserslautern, ErwinSchrödinger-Str. 44, 67663 Kaiserslautern, Germany.

*Email: $\underline{\text { hans.hasse@mv.uni-kl.de }}$

Keywords: polymer aggregation, thermoresponsive polymers, hydrogels, umbrella sampling, potential of mean force, molecular dynamics simulations, simulation method.

\begin{abstract}
Studying equilibrium properties of polymers in solution by atomistic simulations is a challenging task as the available computation time is often not sufficient to ensure representative sampling of the phase space. One approach to tackle this problem is to create a simulation scenario which is simple enough to enable adequate sampling of equilibrium states while it retains the essential parts of the physics of the polymer in solution. In this work, we present and test such a scenario, which is designed for studying whether a given polymer will aggregate or dissolve in a given solvent. Two periodic polymer molecules are simulated in the explicit solvent. The distance $d$ between the polymer chains lends itself as an order parameter so that advanced sampling techniques, such as umbrella sampling, can be applied easily. A state corresponding to dissolved polymers (large $d$ ) and a state corresponding to aggregated polymers (small $d$ ) can be defined. The scenario misses the intramolecular collapse of the single chain, but it retains full atomistic detail regarding the polymer-solvent and the intermolecular polymer-polymer interactions. The
\end{abstract}


thermoresponsive behavior of PNIPAM in water is studied with the new scenario, and it is shown that quantitative predictions of the experimental equilibrium data can be obtained after adjusting a single state-independent parameter in the force field.

\section{Introduction}

Polymer solutions occur in many technical and natural processes. Therefore, it is fundamental to know whether a given polymer will aggregate or dissolve in a given solvent at a given temperature and pressure. Using atomistic simulations for predicting this is attractive. Furthermore, atomistic simulations yield insight into the nature of the phenomena that leads to the macroscopic behavior of the solutions.

The results of atomistic simulations depend on the force field that is applied. We acknowledge that suitable force fields for describing thermodynamic properties of polymer solutions are often not available. In the following, it is assumed that the force field is given. It is just noted that force fields that are taken off the shelf from the literature generally do not describe thermodynamic properties of polymer solutions quantitatively. In the present paper, a new method for exploring results from a given force field is presented. The problem that is addressed here, the determination of equilibrium properties of polymer solutions by atomistic simulations, is a formidable task. In the first step, a simulation scenario has to be chosen. This is far from trivial. The most realistic scenario is one in which many polymer chains move freely in the solvent, and the number of polymer chains and solvent molecules is chosen in such a way that they match the macroscopic polymer concentration. Even for moderately large polymer chains, this typically leads to a prohibitively large phase space that cannot be sampled adequately by atomistic simulations due to limitations of computational power.

There are different ways to tackle this problem: (a) brute force simulations with very large simulation times [1,2], (b) advanced sampling techniques [3,4], (c) coarse-graining modeling of the polymer [5], (d) implicit or coarse-grained solvents [6,7]. In the present work, we aim at exploiting the high potential of all-atom or united-atom force fields so that only (a) and (b) are considered. Unfortunately, even when (a) and (b) are reasonably utilized and suitably combined, it may still be impossible to sample the phase space reliably for the scenario described above. Hence, the problem has to be addressed from the side of 
the scenario. The most common simplified scenario for studying the quality of the solvent is to use a single polymer chain and to monitor whether it collapses or not. Obviously, only intramolecular polymer-polymer interactions are considered in this scenario while the intermolecular polymer-polymer interactions are entirely neglected. Unfortunately, this simplified scenario also suffers from the same problems as the more realistic scenario discussed above, i.e., the phase space often turns out to be too large to be sampled reliably. A more detailed discussion of this is given at the end of this section for $\mathrm{N}$-isopropylacrylamide (NIPAM) polymer chains. The simulation problems encountered in studies of NIPAM motivated the present work, in which a new simple scenario for investigating the aggregation behavior of the polymer in a solvent by atomistic equilibrium simulations was developed.

In the new scenario, we use two periodic polymer molecules that span from one side of the simulation box to the side vis-à-vis. The arrangement is such that no intramolecular collapse occurs and the center of mass of the polymers stays close to the backbone. In direct simulations, the centers of the polymer molecules are furthermore restrained in such a way that both move within a common collision plane. This drastically reduces the phase space that has to be sampled so that studies of equilibria become feasible. Furthermore, the distance $d$ between the centers of the two polymer chains lends itself as an order parameter so that advanced sampling techniques, such as umbrella sampling, can be applied readily. This technique enables calculations of the free energy of the system as a function of $d$. A state corresponding to the dissolved polymer (large numbers of $d$ ) and a state corresponding to aggregated polymers (small numbers of $d$ ) can be defined. Therefore, the transition between these well-defined states can be monitored.

We acknowledge that this scenario is a bold simplification. It misses the intramolecular collapse of the single polymer chain. However, this is not more or less bold than using a single polymer chain and neglecting the intermolecular aggregation. Nevertheless, the latter has the disadvantage that it does not solve the sampling problem. The present two-chain scenario retains full atomistic detail regarding the polymer-solvent and the intermolecular polymer-polymer interactions. This reflects some important aspects of the collapse, for instance, the rearrangement of the side chains upon polymer-polymer contact. 
We claim that this simple scenario gives a good indication whether for a given force field the polymer will be dissolved or aggregated in a given solvent in equilibrium. No strict proof of this claim can be given, but the usefulness of the scenario can be demonstrated. This is done here as described in more detail below.

Some theoretical arguments support the claim. In the following, the change of the free energy of the system upon a transition between the aggregated state and the one in which the individual polymer chains are dissolved is considered. The new scenario retains atomistic resolution regarding all relevant interactions (polymer-solvent, solvent-solvent, and polymer-polymer). Hence, the enthalpic contributions are reasonably covered by the new scenario. However, folded configurations of the backbone could lead to favorable interactions that are not explicitly described by the two-chain scenario. This affects the enthalpy term. In addition, there is more concern about the entropic contributions because in the new scenario the intramolecular collapse is hindered. Nevertheless, the new scenario also captures the influence of the polymer's solvent shell and the release of solvent molecules from that shell upon aggregation. It is well known that this is the main part of the entropic contribution in many situations. The part of the entropic contributions that is neglected here is the one related to the intramolecular conformation change of the polymer chain, which is, e.g., also neglected in Flory's theory.

As a showcase for demonstrating the usefulness of the new scenario, the thermoresponsive behavior of poly(N-isopropylacrylamide) (PNIPAM) in water was studied. Thermoresponsive polymers abruptly change a physical property in response to variations in the temperature. Over the past decades, thermoresponsive polymers have been the subject of intense research due to their potential application in drug delivery, tissue engineering, biosensors, etc. [8-10] The most widely studied thermoresponsive polymer is PNIPAM. It has a lower critical solution temperature (LCST) in water of $305 \mathrm{~K}$ [11-13], i.e., above that temperature PNIPAM and water show a liquid-liquid phase split, i.e., water is a good solvent for PNIPAM at low temperatures, but a poor solvent at high temperatures. In many cases, PNIPAM is used as a hydrogel, in which the polymer chains are crosslinked. Cross-linking does not change the thermoresponsive behavior of PNIPAM substantially.[11] Below the LCST, the hydrogel is swollen and retains a significant amount of water. Above the LCST the hydrogel is collapsed, and the water is released. On 
the molecular scale, the process involves a transition of PNIPAM chains from an extended, random coil conformation to a compact, random globule conformation.[13-15]

Molecular dynamics (MD) simulations with all-atom or united atom models have played an important role in understanding the thermoresponsive behavior of PNIPAM.[11,16-21] The most common scenario that is used in these studies is the simulation of a single 30mer$\mathrm{N}$-isopropylacrylamide (30mer-NIPAM) chain in explicit water.[11,22,23] However, with reasonable computational effort, this scenario can presently be studied for a few hundred nanoseconds. It has become clear recently that this does not enable a sufficient sampling of the phase space. Hence, no reliable information on the equilibrium state as a function of temperature can be obtained with that scenario.[1,2] In previous works, our group has used that scenario for studying the thermoresponsive behavior of PNIPAM in water.[11,16] We demonstrate in Appendix A that also our own simulations were not long enough to yield reliable information on the equilibrium.

The unsatisfactory sampling in simulations of the single oligomer scenario has two main reasons. First, atomistic MD simulations use a time step of the order of femtoseconds. Routinely feasible simulations with millions of time steps lead to simulation times in the nanosecond scale. In molecular simulation studies of the 30mer-NIPAM in water, only a few coil-to-globule transitions can be observed even for very long simulation times, such as $300 \mathrm{~ns}$ [2] or $1000 \mathrm{~ns}$.[1] However, a large number of atomistically different coil-to-globule and globule-to-coil transitions must be observed to make reliable statements about equilibrium properties of that transition. Therefore, there is a large gap between the simulation time required to obtain equilibrium properties and the time scale that is presently accessible by direct simulations. For PNIPAM, the experimental relaxation time to obtain a thermodynamically stable globule state chain from a coiled state is found to be of the order of milliseconds.[24,25] The corresponding information for a 30mer-NIPAM is not available. Second, the usual simulation scenario only relies on intramolecular collapse. Thus, the simulation time required to observe the coil-to-globule transition is limited by the by the rearrangement of many internal degrees of freedom. Thereby, the oligomer must overcome conformational energy barriers that cause a complex energy landscape with many local minima in which the simulation can get trapped. 
The present scenario focusses on intermolecular collapse. It is known from experiments that intermolecular collapse of PNIPAM plays an important role in the thermoresponsive behavior in both dilute and concentrated solutions.[13,26] Dynamic and static light scattering experiments show that even in diluted solution, during a heating cycle, PNIPAM chains undergo first a contraction without fully collapsing before intermolecular collapse leads to the globule state.[13] Recently, by using small-angle neutron scattering, it has been found that the collapse of PNIPAM is driven by chain-chain interaction, while the radius of gyration of single molecules decreases only slightly.[26] A pure intramolecular collapse of PNIPAM can only be observed in extremely diluted solutions $(<5 \mu \mathrm{g} / \mathrm{mL})$.[24,27-29]

In the following, the two-chain simulation scenario is presented. Then, its application in studying the thermoresponsive behavior of PNIPAM in water is discussed. The force field for the PNIPAM + water system and the simulation set up for that application are specified. In that study, the two-chain simulation scenario is used in two ways. First, direct molecular dynamics (MD) simulations are carried out. It is shown that, despite the simplicity of the new scenario, it is difficult to obtain reliable information on the equilibrium from such direct simulations. This strongly underpins the statement that there is no hope to retrieve such information from scenarios which have a much larger phase space, for instance, that in which a 30mer-NIPAM chain moves freely in water. Second, Umbrella sampling was applied for calculating the Potential of Mean Force (PMF) along the transition path, which is feasible with acceptable computational effort. PMF profiles $G(d)$ were determined for PNIPAM in water at temperatures between 280 and $360 \mathrm{~K}$ using the OPLS/AA + SPC/E force field with standard mixing rules, as it has been applied in several previous studies, including our own $[11,16]$. Contrarily to earlier statements in the literature [11], it is shown here that this force field yields demixing of PNIPAM and water in the entire studied temperature range, i.e., no lower critical solution temperature LCST is found. This supports a recent statement of Leonhard and co-workers [2] that was obtained using direct simulations of short NIPAM chains.

For completeness, it is shown that an LCST can be obtained by a simple modification of the mixing rule. Upon a slight increase in the polymer-solvent dispersive interaction energy, the calculated LCST is in the region in which it is also found in experiments. Also, the experimental numbers for the enthalpy and entropy change observed upon demixing are 
reasonably well predicted. This supports the claim that the simplifications of the two-chain scenario are well chosen. The two-chain scenario should also be useful for predicting the behavior of polymer-solvent systems in other situations, e.g., in studies of co-nonsolvency. But this is a hypothesis that remains to be tested.

\section{Two-chain Scenario}

The two-chain simulation scenario is set up as follows. In the first step, two linear chains of the studied polymer consisting of $N$ monomer units each are relaxed in vacuum. In the present work, $N=15$ was chosen. The minimal chain length is limited by the cutoff radius $r_{c}$. To avoid spurious interactions between periodic images, the chain length has to exceed two times the cutoff radius. The two chains are inserted in a rectangular simulation box with their backbones parallel to the $z$-axis. The box length in $z$-direction $l_{\mathrm{z}}$ is chosen such that it equals the length of the relaxed chains, so that the chains span from one side of the box to the side vis-à-vis, see Figure 1. Periodic boundaries are used so that the chains cannot collapse during the simulation as long as $l_{\mathrm{z}}$ is not changed. Information on the choice of the other two box lengths $l_{\mathrm{x}}$ and $l_{\mathrm{y}}$ is given below. After the insertion of the two polymer chains, the simulation box is filled with pre-equilibrated water.

To further reduce the configuration space in direct MD, the movement of the polymer chains in the box is restrained such that their centers of mass move in a collision plane. That plane is marked red in Figure 1 and is perpendicular to the $y$-axis. Technically, a harmonic potential is applied to the $y$-component of the vector connecting the centers of mass of both chains such that the $y$-component of the inter-chain distance $d$ fluctuates around zero. The collision plane itself can move in the $y$-direction in the simulation box when both chains move together in that direction. But the two chains can move independently and freely in both the $x$ and the $z$ directions. This enables a 1-dimensional collapse of the chains.

The distance $d$ between the centers of mass of the chains is a natural order parameter for the present simulation scenario. It is dominated by the distance in $x$-direction whereas in the other two directions only small fluctuations occur. Those in the $z$-direction are not taken into account in the calculation of the distance, i.e., $d$ measures the distance in the $x, y$-plane. An "aggregated state" and a "dissolved state" of the polymer chains are defined by two threshold values: for $d<d^{*}$ the polymer chains are considered to be aggregated, for $d>d^{* *}$ 
they are considered to be dissolved. States with $d$ between these thresholds belong to the transition region. Choosing numbers for $d^{*}$ and $d^{* *}$ is somewhat arbitrary, but can be based on physical arguments which depend on the nature of the polymer and the polymerpolymer interactions. Simple geometrical arguments can be used, such as considering the length of side chains of the polymer, but the choice can also be based on information obtained from the simulation run, e.g., on certain site-site radial distribution functions or the potential of mean force $\mathrm{G}(d)$, as discussed below.

The simulation box size $l_{\mathrm{x}}$ is chosen to be somewhat larger than $d^{* *}$ to enable sampling of the dissolved state. The box length $l_{\mathrm{y}}$ must be large enough to avoid direct interactions between a polymer chain and its solvent shell with its periodic image.

The system containing the two polymer chains and the solvent is equilibrated first in the $N V T$ ensemble and then in the NPT ensemble. The production runs are carried in the NPT ensemble. Since periodic molecules are bonded across the simulation box, isotropic pressure coupling fails to rescale the box dimensions. Therefore, semi-isotropic pressure coupling has to be employed. This may lead to a collapse of the simulation box, i.e., to a decrease of $l_{\mathrm{z}}$ below $2 r_{\mathrm{c}}$. The simplest way to avoid this would be to use a barostat which keeps the box length $l_{\mathrm{z}}$ constant. However, this method would give a poor control of the pressure in the plane that is perpendicular to the z-axis. Thus, a simple workaround was used, which is described in the Supporting Information. 


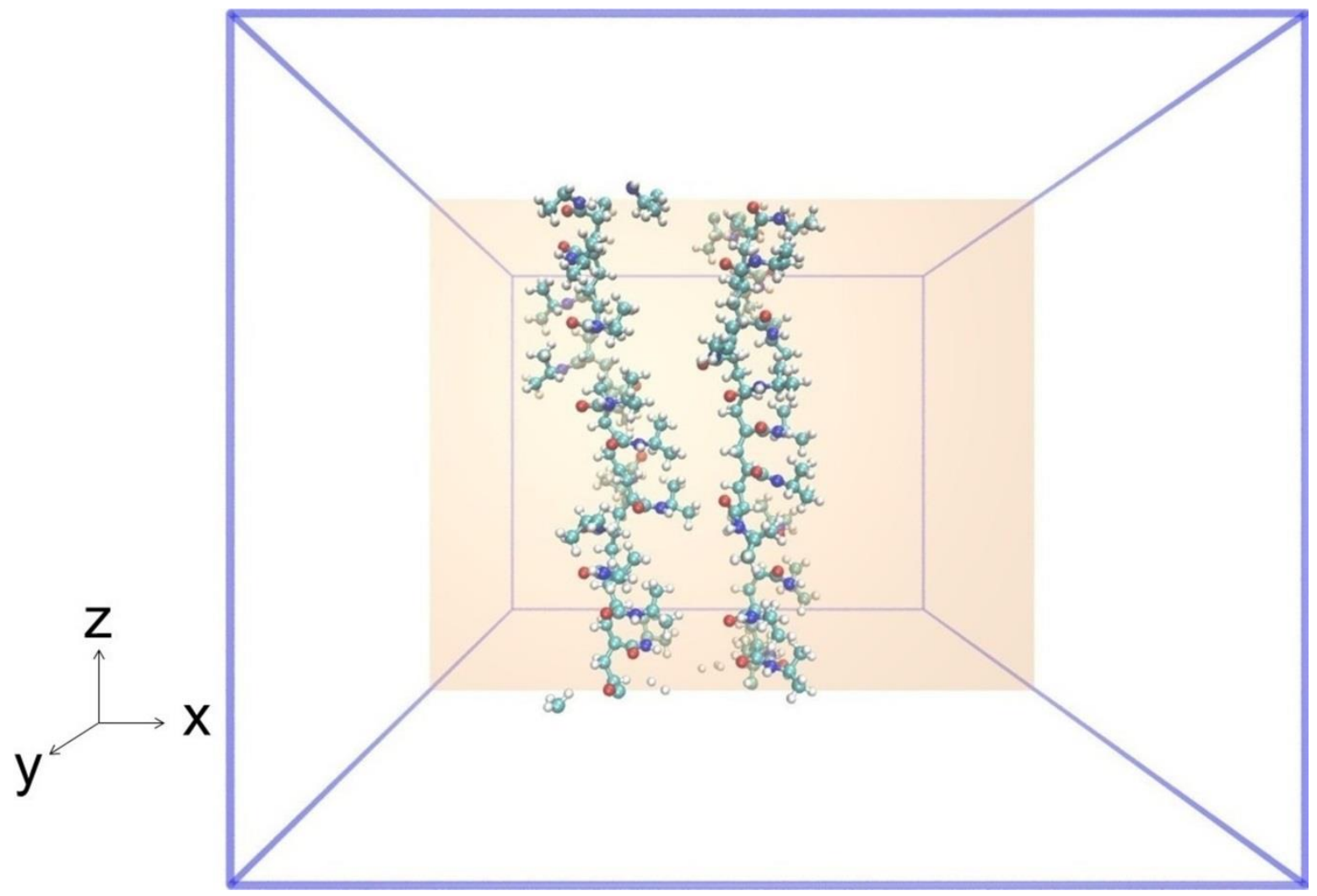

Figure 1. Two-chain simulation scenario illustrated by a snapshot of a simulation of PNIPAM in water. Water molecules are not shown for clarity. The semitransparent red surface indicates the collision plane to which the centers of mass of both PNIPAM molecules are restrained in direct MD. The plane can move backward and forward in the direction of the $y$-axis. Carbons are depicted in green, oxygens in red, nitrogens in blue, and hydrogens in white.

\section{Application to PNIPAM in Water}

\subsection{Force field}

PNIPAM molecules were modeled by the OPLS/AA[30] force field, and water molecules by the SPC/E[31] model, as suggested previously.[11] The PNIPAM molecules used in this work are syndiotactic. The force field parameters are given in the Supporting Information.

A modified Berthelot mixing rule was applied to calculate the polymer-water LJ energy parameters $\left(\varepsilon_{\mathrm{pw}}\right)$, 


$$
\varepsilon_{\mathrm{pw}}=\xi \sqrt{\varepsilon_{\mathrm{pp}} \varepsilon_{\mathrm{ww}}},
$$

where $\xi$ is a state-independent parameter, which can be employed to adjust the interactions between the LJ sites of the polymer (p) and water (w). First, $\xi$ was set to 1 to evaluate the unmodified force field as it was used in Ref. [11], and then it was increased to 1.10 to favor polymer-water interactions. The last value was obtained from preliminary tests. The primary goal of this modification is not to reproduce the experimental LCST of PNIPAM accurately, but merely to demonstrate the capability of the two-chain simulation scenario to describe such phenomena.

According to the recommendations for the OPLS/AA force field, a geometric-mean mixing rule is also used for the LJ size parameters describing the interactions between $\mathrm{LJ}$ sites of the polymer and water

$$
\sigma_{\mathrm{pw}}=\sqrt{\sigma_{\mathrm{pp}} \sigma_{\mathrm{ww}}} .
$$

For the polymer-polymer interaction, unmodified geometric-mean mixing rules were applied to determine mixed Lennard-Jones parameters.

\subsection{Simulations}

All simulations were performed with Gromacs 5.0.[32] Two types of simulations were carried out, direct MD simulations and MD simulations with umbrella sampling.

Two PNIPAM chains of 15 monomers were used, leading to an initial simulation box length in the z-direction of $l_{\mathrm{z}}=3.785 \mathrm{~nm}$, as described in Appendix B. In the other dimensions, the initial simulation box lengths are $l_{\mathrm{x}}=5.0 \mathrm{~nm}, l_{\mathrm{y}}=5.0 \mathrm{~nm}$. The resulting number of water molecules in the box is 2883 . For an illustration, see Figure 1. Results from a systematic study of the effect of the number of monomers per chain and the initial size of the box in the $x$ and $y$ directions are presented in the Supporting Information. The force constant of the harmonic potential which is used to keep the two polymer chains in the collision plane was set to $7000 \mathrm{~kJ} \mathrm{~mol}^{-1} \mathrm{~nm}^{-2}$, which was obtained from preliminary studies. The threshold values for the order parameter were chosen to be $d^{*}=1.2 \mathrm{~nm}$ and $d^{* *}=2.2 \mathrm{~nm}$. The cut-off radius was $1.4 \mathrm{~nm}$. The length of the side chains of PNIPAM is about $0.4 \mathrm{~nm}$. Hence, a rough estimate indicates that for $d>(0.4+0.4+1.4) \mathrm{nm}=2.2 \mathrm{~nm}$ there are no polymer-polymer interactions. In fact, the simulation results show that these interactions are already very weak at distinctly smaller numbers of $d$. Using the same type 
of argument, we deduce that the backbones interact for $d<1.4 \mathrm{~nm}$, so that for $d<d^{*}=1.2$ $\mathrm{nm}$ there are strong interactions not only between the side chains but also between the backbones. These choices of $d^{*}$ and $d^{* *}$ are also confirmed by the results for the potential of mean force $G(d)$ presented below.

The system is equilibrated first in the canonical (NVT) ensemble for 100 ps and then in the NPT ensemble for $250 \mathrm{ps}$. The production runs are carried out in the NPT ensemble for 120 ns. Simulations were carried out for temperatures between 280 and $360 \mathrm{~K}$ in intervals of $20 \mathrm{~K}$. The pressure was set to 1 bar. Semiisotropic coupling was used to keep the pressure constant. As a result, the size of the box in the $z$-direction can change independently of that in the $x$ and $y$ directions. The intramolecular collapse was avoided by applying restraints on specific atoms in the backbone of the chains. Details are given in Appendix B.

\subsection{PMF from umbrella sampling}

The potential of mean force $G(d)$ is determined from the probability distribution function $P(d)$, which describes the probability of finding the system in states in an infinitesimal interval around $d$. As direct simulations are unfeasible for converging $P(d)$ (see below), umbrella sampling [33-35] was applied. In umbrella sampling, the system is restrained in successive configurations, called umbrella windows, along the transition path. Thus, favorable and unfavorable states on the transition path can be satisfactorily sampled. A harmonic potential $W(d)$ is applied to restrain the system to specific sections of the path. The relation between the biased probability distribution $P^{\mathrm{b}}(d)$ and $G(d)$ is given by

$$
G(d)=-R T \ln \left[P^{\mathrm{b}}(d)\right]-W(d)+G_{0},
$$

where $G_{0}$ is used for normalization. $G(d)$ was obtained here using 34 overlapping windows in which $d$ was varied between 0.8 and $2.2 \mathrm{~nm}$. The weighted histogram analysis method WHAM[36] was used to reconstruct $G(d)$ along the path as implemented in Gromacs.[37] The normalization was chosen so that $G=0$ for the maximal distance $d=2.2 \mathrm{~nm}$. Technical details of the simulations and calculation of the PMF are given in Appendix C. 


\section{Results and discussion}

\subsection{Direct simulations (unmodified polymer-water mixing rule, $\xi=1$ )}

Two independent simulations with different initial configurations, an aggregated state $\left(d_{\text {ini }}=0.9 \mathrm{~nm}\right)$ and a dissolved state $\left(d_{\text {ini }}=1.8 \mathrm{~nm}\right)$, were conducted at $280 \mathrm{~K}$ using the twochain scenario. The results are shown in Figure 2. For both simulations, the polymerpolymer distance $d$ fluctuates between 0.86 and $2.5 \mathrm{~nm}$. The upper limit of $d$ results from the size of the simulation box in the $x$-direction $(\sim 5 \mathrm{~nm})$ and the periodic boundary conditions while the lower limit results from polymer-polymer repulsion. For the simulation with $d_{\text {ini }}=0.9 \mathrm{~nm}$, the PNIPAM chains remain aggregated until approximately $t$ $=16 \mathrm{~ns}$, after this time they move into the dissolved state for $5 \mathrm{~ns}$. Then, the chains recover the aggregated state again and stay in this configuration for the rest of the simulation time of $120 \mathrm{~ns}$. For the simulation with $d_{\mathrm{ini}}=1.8 \mathrm{~nm}$, significant fluctuation in the polymerpolymer distance is observed. During the initial $100 \mathrm{~ns}$, the PNIPAM chains are mainly in the dissolved state, but several collisions with $d<1.4 \mathrm{~nm}$ occur approximately every $10 \mathrm{~ns}$ before the molecules aggregate permanently at $t=94 \mathrm{~ns}$. Movies of both simulations (V1 and V2) are supplied with the Supporting Information. Even though there seems to be a preference for the aggregated state at the studied conditions, the results are ambiguous: the simulation with $d_{\text {ini }}=1.8 \mathrm{~nm}$ remained most of the time in the dissolved state whereas the simulation $d_{\text {ini }}=0.9 \mathrm{~nm}$ stayed most of the time in the aggregated state. 


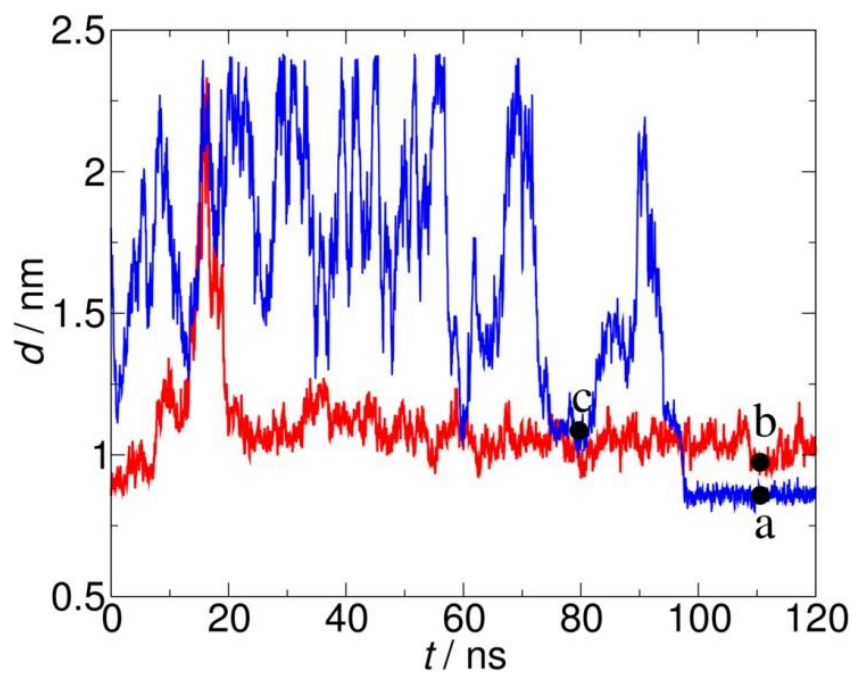

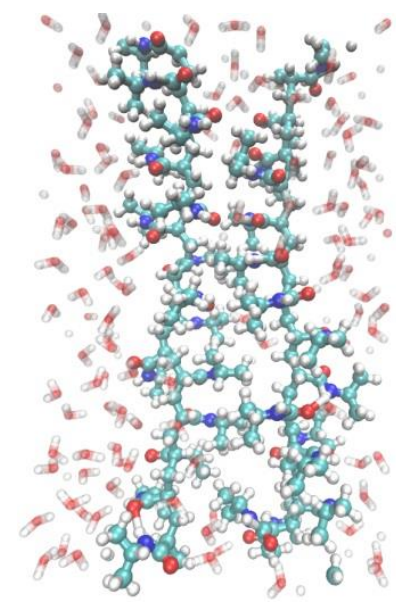

(a)

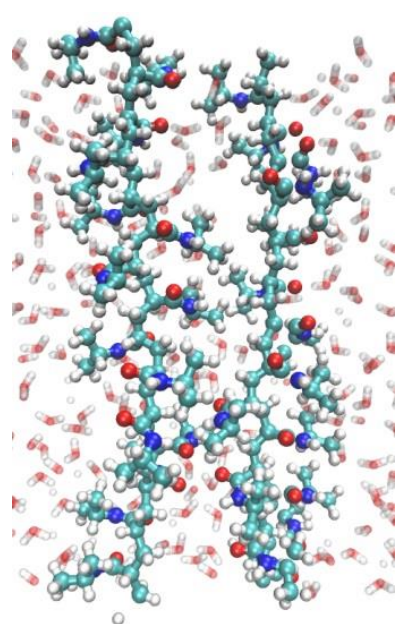

(b)

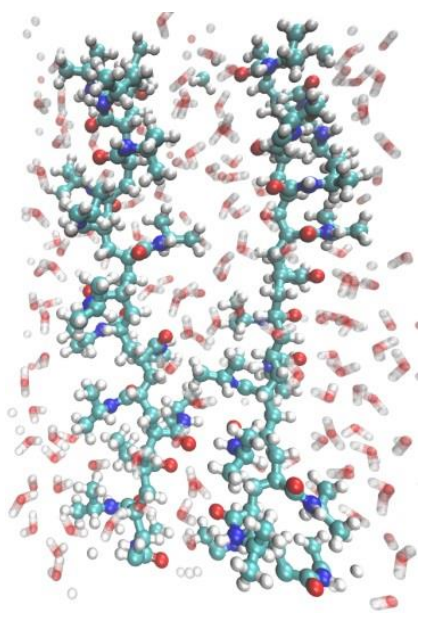

(c)

Figure 2. Simulation results for PNIPAM in water at $280 \mathrm{~K}$ obtained with the two-chain scenario $(\xi=1.0)$. Top: chain-chain distance as a function of simulation time at $280 \mathrm{~K}$. The simulations were started from different configurations, $d_{\text {ini }}=0.9 \mathrm{~nm}$ (red line) and $d_{\text {ini }}=1.8$ $\mathrm{nm}$ (blue line). Bottom: Snapshots of favorable conformations: (a) fully aggregated $(d=$ $0.87 \mathrm{~nm})$; (b) partially aggregated $(d=0.96 \mathrm{~nm})$, and $(\mathrm{c})$ water-mediated aggregation $(d=$ $1.12 \mathrm{~nm}$ ). Only water molecules in the layer of $1 \mathrm{~nm}$ around PNIPAM chains are depicted.

Two different aggregated states, one with $d=0.87 \mathrm{~nm}$ and the other with $d=0.96 \mathrm{~nm}$, are attained at the end of the simulation runs, and both seem to be favorable, cf. Figure 2. Snapshots of the corresponding configurations are shown in the bottom of Figure 2. For $d=$ 
$0.87 \mathrm{~nm}$ (panel (a), fully aggregated state) most of the side chains are in direct contact with the side chains of the other PNIPAM molecule, whereas for $d=0.96 \mathrm{~nm}$ (panel (b), partially aggregated state) there are fewer side-chain side-chain contacts. Furthermore, Figure 2 shows that there is a third particularly favorable state with $d=1.12 \mathrm{~nm}$, which corresponds to a water-mediated aggregation, panel (c).

The transition between the aggregated and the dissolved state can be observed in both directions within a reasonable simulation time. In the traditional single-oligomer scenario, generally not more than one transition is obtained for similar simulation time (see Supporting Information), and a much larger simulation volume is needed.[2,11] Still, even with the two-chain scenario, it is difficult to obtain unambiguous results regarding the equilibrium state when direct simulations are applied, cf. Figure 2.

\subsection{Umbrella sampling (unmodified polymer-water mixing rule, $\xi=1$ )}

The two-chain simulation scenario was used to determine the potential of mean force $G(d)$ by umbrella sampling as described above. The results for $\xi=1$ and $280 \mathrm{~K}$ are presented in Figure 3. They show that the free energy barrier that has to be overcome for the transition from the dissociated to the aggregated state is only about $2 \mathrm{~kJ} \mathrm{~mol}^{-1}$ and therefore of the order of $R T$. Despite this comparatively small thermodynamic barrier in the two-chain simulation scenario, it takes almost $100 \mathrm{~ns}$ for that transition to happen in direct simulations (Figure 2), which shows that there are important kinetic limitations even in the simple scenario used here. On the other hand, the free energy barrier which has to be overcome for the transition from $d=0.92 \mathrm{~nm}$ to $d=2.20 \mathrm{~nm}$, i.e., going from the aggregated state to the dissolved state, is about $14 \mathrm{~kJ} / \mathrm{mol}$, corresponding to about $6 R T$. Together with the additional kinetic limitations, this explains why in direct simulations the disentanglement is hard to observe, even with the simple scenario.

The global minimum of $G(d)$ indicates the most stable conformation. At $280 \mathrm{~K}$, the global minimum of $G(d)$ is at $d=0.92 \mathrm{~nm}$ (Figure 3), i.e., in an aggregated state. The potential of mean force was also determined at 300, 320, 340, and $360 \mathrm{~K}$. The results are presented in the Supporting Information. They show that the tendency of PNIPAM to aggregate increases with the temperature. So, there is not dissolved-aggregated transition for this force field for the studied temperatures. Therefore, it can be concluded that the 
OPLS/AA + SPC/E force field is unable to reproduce the temperature-dependent transition of PNIPAM. Recently, a similar conclusion was reported based on simulations of the phase transition of short oligomers.[2]

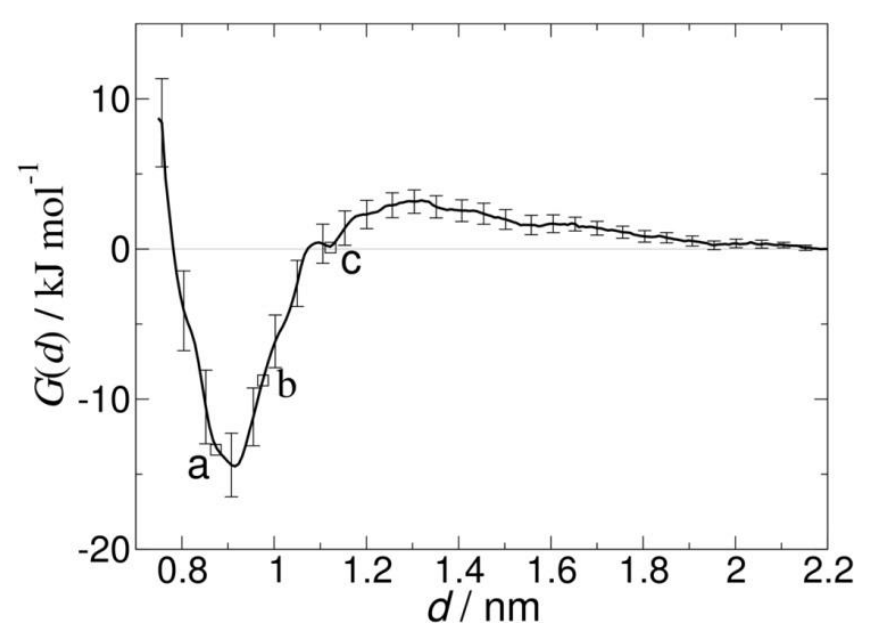

Figure 3. Potential of mean force of the transition between the dissolved and the aggregated state of PNIPAM in water at $280 \mathrm{~K}$. The results were obtained with the twochain scenario using umbrella sampling $(\xi=1)$. The points a-c correspond to favorable configurations observed in the direct simulations shown in Figure 2, (a) fully-aggregated, (b) partially-aggregated, and (c) water-mediated conformations. The error bars were obtained from the simulation runs in the individual windows.

The direct simulations generally do not lead to the global minimum in the PMF. The favorable conformations found in the direct simulations described in Section 4.1 (fullyaggregated, partially-aggregated, and water-mediated, cf. Figure 2) are plotted in Figure 3 by indicating the respective values of $d$. Neither of these conformations corresponds to the global minimum of the PMF, so they are all metastable conformations. However, it can be seen that conformation (a) is close to the global minimum.

\subsection{Umbrella sampling (modified polymer-water mixing rule, $\xi=1.1$ )}

The results discussed above show that the unmodified OPLS/AA + SPC/E force field does not predict the temperature dependent transition of PNIPAM, i.e., at all studied temperatures PNIPAM chains were found to be aggregated. To demonstrate the ability of the two-chain scenario to study the transition, a modified force field was used, which was chosen based on results of a preliminary study. In the modified force field, the polymer- 
water dispersion interaction was increased by $10 \%$, i.e., $\xi=1.10$. As the polymer-solvent dispersive attraction increases, the solvent becomes a better solvent for the polymer so that the dissolved-aggregated transition occurs at higher temperature. Figure 4 shows results for the potential of mean force $G(d)$ obtained using $\xi=1.1$ for temperatures between 280 and $360 \mathrm{~K}$. By comparing $G(d)$ obtained at $280 \mathrm{~K}$ and $\xi=1.0$ (Figure 3 ) and $\xi=1.1$ (Figure 4), it can be seen that increasing $\xi$ leads to an important increase in $G(d)$ for all values of $d$. For $\xi=1.1$ and $280 \mathrm{~K}, G(d)$ still has a minimum at around $d=0.9 \mathrm{~nm}$, but that minimum is no longer the global minimum. The global minimum is now the dissolved state obtained for a larger distance $d$. At $280 \mathrm{~K}$ and $\xi=1.1$ (Figure 4), the energy barrier which has to be overcome for the transition from the metastable aggregated state at about $d=0.9 \mathrm{~nm}$ to the stable dissolved state at large $d$ is about $3 R T$.

Increasing the temperature shifts the global minimum in the potential of mean force $G(d)$. For all studied temperatures, there is a minimum of $G(d)$ at about $d=0.9 \mathrm{~nm}$, corresponding to an aggregated state. But only for temperatures above about $300 \mathrm{~K}$, that minimum is the global minimum, i.e., the equilibrium configuration changes from dissolved to aggregated at about $300 \mathrm{~K}$, as observed in experiments. At $300 \mathrm{~K}, G(d)$ has two minima in which $G(d)$ is close to zero, and the system fluctuates between these two states, hindered by a thermodynamic barrier of about $3 R T$. 


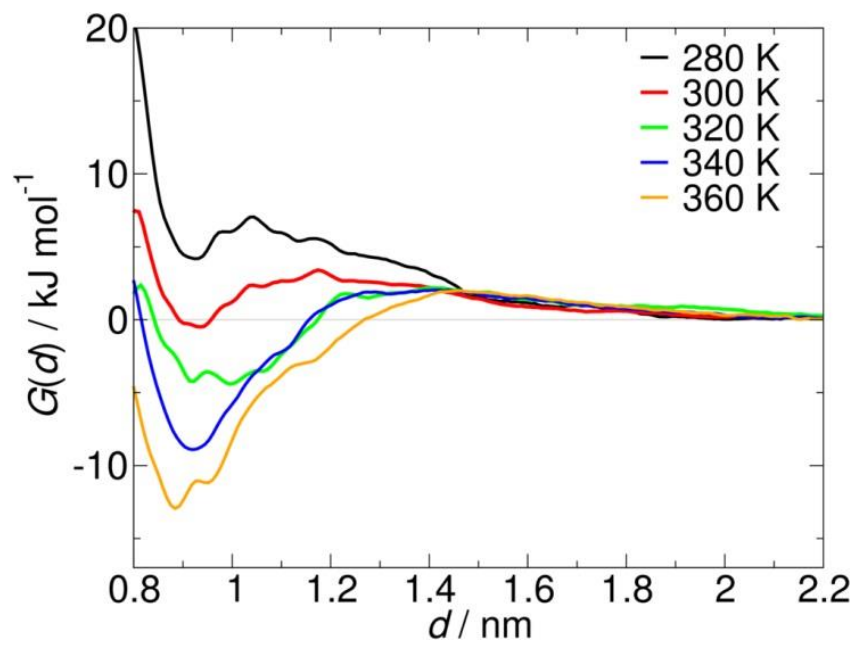

Figure 4. Potential of mean force of the transition between the dissolved and the aggregated state of PNIPAM in water for different temperatures. The results were obtained with the two-chain scenario using umbrella sampling $(\xi=1.1)$.

The Gibbs energy of the dissolved state $G_{\mathrm{d}}$ is zero by definition here. The Gibbs energy of the aggregated state $G_{\mathrm{a}}$ can be obtained by integrating $G(d)$ as follows [38]:

$$
G_{\mathrm{a}}=-R T \ln \frac{\int_{d_{1}}^{d_{2}} \exp \left(-\frac{G(d)}{R T}\right) \mathrm{d} d}{d_{2}-d_{1}} .
$$

In the present work, the upper bound used for calculating $G_{\mathrm{a}}$ is $d_{2}=d^{*}=1.2 \mathrm{~nm}$. As no configurations were observed below $0.8 \mathrm{~nm}, d_{1}$ is set to $0.8 \mathrm{~nm}$. As $G_{\mathrm{d}}$ is zero, the number for $G_{\mathrm{a}}$ is also the number obtained for the change of the Gibbs energy upon the dissolvedto-aggregated transition $\Delta G_{\mathrm{d} \rightarrow \mathrm{a}}$. The results for $\Delta G_{\mathrm{d} \rightarrow \mathrm{a}}$ obtained from the evaluation of the potential of mean force $G(d)$ at different temperatures are shown in Figure 5. The error bars reported for $\Delta G_{\mathrm{d} \rightarrow \mathrm{a}}$ is the difference obtained from integrating Equation 4 using $G(d)$ and $G(d)+\Delta G(d)$, respectively. The uncertainty of the potential of mean force $\Delta G(d)$ was estimated using the bootstrap analysis (see Appendix $\mathrm{C}$ for details).

It can be seen from Figure 5 that $\Delta G_{\mathrm{d} \rightarrow \mathrm{a}}$ changes its sign at about $300 \mathrm{~K}$. Figure 5 also shows the results from a fit of $\Delta G_{\mathrm{d} \rightarrow \mathrm{a}}$ with

$$
\Delta G_{\mathrm{d} \rightarrow \mathrm{a}}=\Delta H_{\mathrm{d} \rightarrow \mathrm{a}}-T \Delta S_{\mathrm{d} \rightarrow \mathrm{a}},
$$

from which the changes of the enthalpy $\Delta H_{\mathrm{d} \rightarrow \mathrm{a}}$ and the entropy $\Delta S_{\mathrm{d} \rightarrow \mathrm{a}}$ per monomer unit of PNIPAM and the transition temperature $T_{\theta}$, at which $\Delta G_{\mathrm{d} \rightarrow \mathrm{a}}$ changes its sign, was 
determined. The resulting number for $T_{\theta}=309 \pm 9 \mathrm{~K}$, which is in good agreement with the experimental data for the LCST of PNIPAM, cf. Table 1. The same holds for $\Delta H_{\mathrm{d} \rightarrow \mathrm{a}}$ and $\Delta S_{\mathrm{d} \rightarrow \mathrm{a}}$ for which the experimental values scatter considerably. The uncertainty in the simulated value of $T_{\theta}$ reported in Table 1 was obtained by fitting $\left(\Delta G_{\mathrm{d} \rightarrow \mathrm{a}}+\Delta \Delta G_{\mathrm{d} \rightarrow \mathrm{a}}\right)$ vs. $T$, where the uncertainty $\Delta \Delta G_{\mathrm{d} \rightarrow \mathrm{a}}$ was taken from the results shown in Figure 5. On the other hand, the uncertainty in $\Delta S_{\mathrm{d} \rightarrow \mathrm{a}}$ and $\Delta H_{\mathrm{d} \rightarrow \mathrm{a}}$ was calculated by taking the largest deviation in the slope and the intercept for all possible lines that pass through the interval defined by $\Delta \Delta G_{\mathrm{d} \rightarrow \mathrm{a}}$. The good agreement with the experimental values obtained after adjusting the mixing rule is not a proof of the assumptions made in the two-chain scenario. However, the good agreement shows that the simplifications are useful to describe the aggregation process from a practical standpoint.

The transition temperature $T_{\theta}$ depends strongly on the choice of $\xi$. Additional simulations were carried out for $\xi=1.15$, which yielded $T_{\theta}=340 \pm 6 \mathrm{~K}$. For details see Supporting Information. 


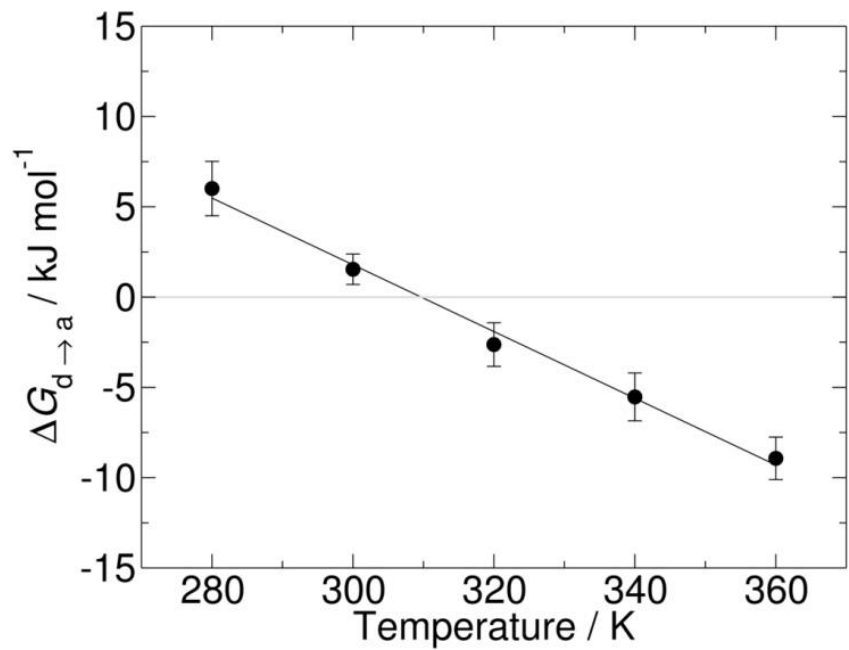

Figure 5. Free energy change of the transition from the dissolved to the aggregated state of PNIPAM in water as a function of the temperature. Results obtained with the two-chain scenario using umbrella sampling $(\xi=1.1)$.

Table 1. Thermodynamic properties of the transition from the dissolved to the aggregated state of PNIPAM in water. Comparison of simulation results obtained with the two-chain scenario using umbrella sampling to experimental data from the literature. The binary interaction coefficient used in the simulations is $\xi=1.1$.

\begin{tabular}{lll}
\hline Property & Simulation & Experiment \\
\hline$T_{\theta} / \mathrm{K}$ & $309 \pm 9$ & $305^{\mathrm{a}}-306^{\mathrm{b}}-307^{\mathrm{c}}-310^{\mathrm{d}}$ \\
$\Delta H_{\mathrm{d} \rightarrow \mathrm{a}} / \mathrm{kJ} \mathrm{mol}^{-1}$ per monomer & $1.9 \pm 0.4$ & $1.8^{\mathrm{b}}-3.8^{\mathrm{c}}-4.4^{\mathrm{d}}-4.8^{\mathrm{e}}$ \\
$\Delta S_{\mathrm{d} \rightarrow \mathrm{a}} / \mathrm{J} \mathrm{K}^{-1} \mathrm{~mol}^{-1}$ per monomer & $6.2 \pm 1.2$ & $6^{\mathrm{b}}-12^{\mathrm{c}}-14^{\mathrm{d}}$ \\
\hline
\end{tabular}

${ }^{\text {a }}$ Swelling of the hydrogel, from Ref. [11].

${ }^{b}$ Differential scanning calorimetry (DSC) of a 20mer-PNIPAM, from Ref. [39].

${ }^{c}$ DSC of a 92mer-PNIPAM, from Ref. [40].

${ }^{\mathrm{d}}$ DSC of PNIPAM with a molar mass of $4.9 \times 10^{4} \mathrm{~g} / \mathrm{mol}$, from Ref. [41].

${ }^{\mathrm{e}}$ DSC of PNIPAM with a molar mass of $3.3 \times 10^{3} \mathrm{~g} / \mathrm{mol}$, from Ref [42]. 


\section{Conclusions}

In this work, a two-chain molecular simulation scenario is presented. It can be used for assessing whether a polymer is aggregated or dissolved in a given solvent based on atomistic force fields. In the scenario, the intermolecular collapse of two periodic polymer chains is monitored.

In atomistic studies of polymer solutions, scenarios with a single, freely moving oligomer are often used. In those studies, the simulation time must be long enough such that the oligomer can undergo intramolecular collapse and visit all relevant conformations. Unfortunately, the phase space of such scenarios is often so large that reliable information on the equilibrium cannot be obtained in acceptable simulation time. For instance, once the single oligomer is collapsed, it is usually impossible to observe its unfolding in the simulation. The sampling problems increase when multiple freely moving chains are used. Hence, for practical reasons, it is hard to assess with the established molecular simulation methods whether a polymer is aggregated or dissolved in a given solvent in equilibrium. The new scenario aims at solving this problem by reducing the phase space which has to be sampled in such a way that essential elements of the atomistic description of the transition of the polymer from the dissolved state to the aggregated state in the solvent are preserved. All interactions are accounted for with atomistic resolution. Only the intramolecular collapse is excluded. This leads to a significant reduction of the phase space so that equilibria can now be monitored. Despite the simplification, essential elements of the collapse are retained, e.g., the release of the solvent from the polymer's solvent shell, the increased number of polymer-polymer contacts, etc. Basically, only the contribution of the folding is neglected. This simplification is assumed to be comparable to other possible sources of error, e.g., deficiencies of force fields.

In the two-chain scenario, the distance of the two polymer chains $d$ is a natural order parameter so that it is particularly suited for applications of advanced sampling techniques which facilitate the calculation of the potential of mean force $G(d)$ from which global and local equilibrium states and energy barriers between these states can be found.

The usefulness of the two-chain scenario is demonstrated using the thermoresponsive behavior of PNIPAM in water as an example. The potential of mean force profiles reveals that the OPLS/AA + SPC/E force field predicts that water is a bad solvent for all studied 
temperatures, which were between 280 and $360 \mathrm{~K}$. Therefore, the binary interaction coefficient $\xi$ in the modified mixing rule for the water-polymer interaction was increased from 1 to 1.1. Using this modified force field, it was demonstrated that the thermoresponsive behavior of PNIPAM in water is described well, i.e., water is a good solvent at low temperatures and a bad one at high temperatures. The transition temperature $T_{\theta}$ as well as the enthalpy and entropy change per monomer unit of PNIPAM were found to be in good agreement with the experimental data. This supports the statement that the simplifications of the scenario are acceptable from a practical standpoint. However, it does not exclude that the result is only favorable due to a cancellation of errors. Nevertheless, the result is encouraging and shows that it is worthwhile to test the new method for more examples.

The new scenario opens the route for assessing the quality of different solvents or for different polymers based solely on atomistic force fields. This includes mixed solvents, solvents containing salts, copolymers, polyelectrolytes, etc. The success or failure of such predictions strongly depends on the force field. The new two-chain scenario also enables improving the force fields for describing polymer-solvent systems.

\section{Appendix A. Criticism of Walter et al. [11]}

The paper of Walter et al. [11] reports experimental and molecular simulation results on the coil-to-globule transition of PNIPAM in water. The molecular simulations were carried out using the single chain scenario with a 30mer-PNIPAM. The length of the production run was $20-40 \mathrm{~ns}$. It is shown in the Supporting Information that this is far too short to sample the equilibrium reliably. Here, the simulation time was extended to $120 \mathrm{~ns}$, but still, no adequate sampling was obtained, see Supporting Information. It is known from the literature that the sampling problems persist even when the simulation time is increased to about 1000 ns.[1] A second problem with the simulations of Walter et al. [11] is that the simulation volume was not large enough to avoid artificial head-tail interactions in some orientations of the stretched chain. This artificially favors the stretched state over the collapsed state. 


\section{Appendix B. MD simulations details}

The system was equilibrated by the following protocol. First, PNIPAM chains were relaxed in vacuum and then with the solvent, using the steepest descent method with a target force of $100 \mathrm{~kJ} \mathrm{~mol}^{-1} \mathrm{~nm}^{-2}$. The initial inter-chain distance $d_{\text {ini }}$ was kept at the desired value during the equilibration steps using a harmonic potential.

The leapfrog integrator was used with a time step of $1 \mathrm{fs}$. Non-bonded interactions were truncated using a cutoff radius of $1.4 \mathrm{~nm}$. Tail corrections were implemented for the potential energy and pressure. The neighbor list was updated every ten integration steps. Electrostatic interactions were calculated by the PME method.[43] The grid spacing was fixed at $0.12 \mathrm{~nm}$, and the interpolation order was carried out up to fourth. The cutoff radius for the real space sum was set to $1.4 \mathrm{~nm}$. Periodic boundary conditions were applied in all dimensions of space. The temperature was established by the V-scaling thermostat[44] with a time coupling constant of $0.1 \mathrm{ps}$. The Berendsen barostat was used to set the pressure at 1 bar.[45] The pressure coupling constant was $0.5 \mathrm{ps}$ and the compressibility $4.510^{-5} \mathrm{bar}^{-1}$. Semi-isotropic pressure coupling was employed, i.e., the z-lattice vector of the box was adjusted independently of the $x$ and $y$ vectors. Resulting from the action of the barostat, the simulation box could collapse in the z-direction as a consequence of the intramolecular collapse of the PNIPAM chains. The intramolecular collapse was avoided by restraining the distance between specific pairs of methylene carbons $\left(\mathrm{CH}_{2}\right)$ in the backbone. Specifically, a harmonic potential was applied when the distance between the methylene carbons was lower than the distance in the pre-equilibrated linear configuration. Thus, the harmonic potential only pulls to avoid the intramolecular collapse, but it does not prevent the stretching. Three restraints pairs per chain were applied to allow certain flexibility of the chain. The restrained pairs were the methylene carbons in monomers 1-7, 4-10, and 915. The force constant for the harmonic potential was $20,000 \mathrm{~kJ} \mathrm{~mol}^{-1} \mathrm{~nm}^{-2}$. These constraints are work-arounds that facilitate the NPT simulation with the given barostat. They have no modeling background.

\section{Appendix C. Umbrella sampling and calculation of the potential of mean force}

Umbrella sampling was carried out for the two-chain simulation scenario for a distance $d$ between 0.8 and $2.2 \mathrm{~nm}$. A total of 34 umbrella windows were used. For $d>1.1 \mathrm{~nm}$, the 
central position of the window is on a grid with a spacing of $0.05 \mathrm{~nm}$. For $d<1.1 \mathrm{~nm}$, the grid spacing was $0.025 \mathrm{~nm}$.

The force constant of the harmonic potential for restraining $d$ was $7000 \mathrm{~kJ} \mathrm{~mol}^{-1} \mathrm{~nm}^{-2}$. This value was obtained by trial and error. Two factors were considered to get the force constant: maximizing the overlap of umbrella histograms of successive windows and keeping a reasonable number of windows.[46] As an example, an umbrella histogram is shown in the Supporting Information.

The equilibration and production simulations were carried as follows. First, the pull algorithm in Gromacs was applied to generate the initial configuration for a given $d$.[32] Then, for each window, two successive equilibration simulations were conducted: the first one in the $N V T$ ensemble for $100 \mathrm{ps,}$ and the second in the $N P T$ ensemble for $750 \mathrm{ps}$. The same simulation conditions and parameters as described in Appendix B were applied. After equilibration, the production run was conducted in the NPT ensemble. Short independent simulations in narrow umbrella windows reduce statistical errors and increase computational efficiency.[46] The independent simulations also avoid the system getting trapped in a given configuration, which is critical in sampling the aggregated state. Thus, for each umbrella window, eight independent production simulations of $3 \mathrm{~ns}$ each were conducted, so the total production time per window was 24 ns. Since 34 windows were defined, the total simulation time used to determine each PMF profile was $816 \mathrm{~ns}$. All runs were started from different initial configurations, and different random seeds were used to assign the velocities.

In production runs, the mean force was recorded every $10 \mathrm{fs}$. The potential of mean force $G(d)$ was calculated along $d$ by using the WHAM in Gromacs.[37] The reference state of the PMF was taken at $2.2 \mathrm{~nm}$, which correspond to the dissolved state. To determine the uncertainty of the PMF profiles, we used the bootstrap method [37] with 200 bootstraps. The PMF was calculated using a running average and did not change after 24 ns per window (for details see Supporting Information).

\section{Nomenclature}

\section{Symbols}

$d \quad$ distance in the $x, y$ plane 


$\begin{array}{ll}G(d) & \text { potential of mean force } \\ P & \text { probability distribution } \\ T & \text { absolute temperature } \\ W(d) & \text { harmonic potential }\end{array}$

\section{Abbreviations}

DSC differential scanning calorimetry

OPLS/AA optimized potentials for liquid simulations / all-atom

PMF potential of mean force

PNIPAM poly(N-isopropylacrylamide)

SPC/E extended simple point charge (water model)

\section{Subscripts}

$\begin{array}{ll}\text { a } & \text { aggregated state } \\ \text { d } & \text { dissolved state } \\ \text { ini } & \text { initial }\end{array}$

\section{Acknowledgment}

The authors thank Juan M. Castillo, Jonathan Walter, and Jadran Vrabec for fruitful discussions. The authors acknowledge financial support from the Reinhart Koselleck Program (HA1993/15-1) of the German Research Foundation (DFG) and the German Federal Ministry of Education and Research (BMBF) within the project TalPas $(01 \mathrm{IH} 16008 \mathrm{~F})$. The present work was conducted under the auspices of the Boltzmann-Zuse Society of Computational Molecular Engineering (BZS). The simulations were carried out on the Elwetritsch at the Regional University Computing Center Kaiserslautern (RHRK) under the grant TUK-TLMV, on SuperMUC at the Leibniz Supercomputing Center (LRZ), Garching, under the grant SPARLAMPE (pr48te), and on Hazel Hen at the High Performace Computing Center, Stuttgart (HLRS), under the grant MMHBF2.

\section{References}

[1] Y. Kang, H. Joo, J.S. Kim, Collapse-Swelling Transitions of a Thermoresponsive, Single Poly(N-isopropylacrylamide) Chain in Water, J. Phys. Chem. B. 120 (2016) 13184-13192. doi:10.1021/acs.jpcb.6b09165. 
[2] V. Boțan, V. Ustach, R. Faller, K. Leonhard, Direct phase equilibrium simulations of NIPAM oligomers in water, J. Phys. Chem. B. 120 (2016) 3434-3440. doi:10.1021/acs.jpcb.6b00228.

[3] R. Day, D. Paschek, A.E. Garcia, Microsecond simulations of the folding/unfolding thermodynamics of the Trp-cage miniprotein, Proteins Struct. Funct. Bioinforma. 78 (2010) 1889-1899. doi:10.1002/prot.22702.

[4] M.L. Galbraith, J.D. Madura, Identifying trends in hydration behavior for modifications to the hydrophobicity of poly(n-isopropylacrylamide), J. Mol. Graph. Model. 78 (2017) 168-175. doi:10.1016/j.jmgm.2017.09.021.

[5] L.J. Abbott, M.J. Stevens, A temperature-dependent coarse-grained model for the thermoresponsive polymer poly(N-isopropylacrylamide), J. Chem. Phys. 143 (2015) 244901. doi:10.1063/1.4938100.

[6] A.J. Schmid, J. Dubbert, A.A. Rudov, J.S. Pedersen, P. Lindner, M. Karg, I.I. Potemkin, W. Richtering, Multi-Shell Hollow Nanogels with Responsive Shell Permeability, Sci. Rep. 6 (2016) 22736. doi:10.1038/srep22736.

[7] D. Mukherji, K. Kremer, Coil-Globule-Coil Transition of PNIPAm in Aqueous Methanol: Coupling All-Atom Simulations to Semi-Grand Canonical Coarse-Grained Reservoir, Macromolecules. 46 (2013) 9158-9163. doi:10.1021/ma401877c.

[8] M.A. Ward, T.K. Georgiou, Thermoresponsive polymers for biomedical applications, Polymers. 3 (2011) 1215-1242. doi:10.3390/polym3031215.

[9] L. Klouda, Thermoresponsive hydrogels in biomedical applications: A seven-year update, Eur. J. Pharm. Biopharm. Off. J. Arbeitsgemeinschaft Für Pharm. Verfahrenstechnik EV. 97 (2015) 338-349. doi:10.1016/j.ejpb.2015.05.017.

[10] M.A.C. Stuart, W.T.S. Huck, J. Genzer, M. Müller, C. Ober, M. Stamm, G.B. Sukhorukov, I. Szleifer, V.V. Tsukruk, M. Urban, F. Winnik, S. Zauscher, I. Luzinov, S. Minko, Emerging applications of stimuli-responsive polymer materials, Nat. Mater. 9 (2010) 101-113. doi:10.1038/nmat2614.

[11] J. Walter, V. Ermatchkov, J. Vrabec, H. Hasse, Molecular dynamics and experimental study of conformation change of poly(N-isopropylacrylamide) hydrogels in water, Fluid Phase Equilibria. 296 (2010) 164-172. doi:10.1016/j.fluid.2010.03.025.

[12] H.G. Schild, Poly(N-isopropylacrylamide): experiment, theory and application, Prog. Polym. Sci. 17 (1992) 163-249. doi:10.1016/0079-6700(92)90023-R.

[13] H. Cheng, L. Shen, C. Wu, LLS and FTIR Studies on the Hysteresis in Association and Dissociation of Poly(N-isopropylacrylamide) Chains in Water, Macromolecules. 39 (2006) 2325-2329. doi:10.1021/ma052561m.

[14] T. Tokuhiro, T. Amiya, A. Mamada, T. Tanaka, NMR study of poly(Nisopropylacrylamide) gels near phase transition, Macromolecules. 24 (1991) 29362943. doi:10.1021/ma00010a046.

[15] F. Zeng, Z. Tong, H. Feng, N.m.r. investigation of phase separation in poly(Nisopropyl acrylamide)/water solutions, Polymer. 38 (1997) 5539-5544. doi:10.1016/S0032-3861(97)00118-3.

[16] J. Walter, J. Sehrt, J. Vrabec, H. Hasse, Molecular Dynamics and Experimental Study of Conformation Change of Poly(N-isopropylacrylamide) Hydrogels in Mixtures of Water and Methanol, J. Phys. Chem. B. 116 (2012) 5251-5259. doi:10.1021/jp212357n. 
[17] Y. Tamai, H. Tanaka, K. Nakanishi, Molecular dynamics study of polymer-water interaction in hydrogels. 1. Hydrogen-Bond structure, Macromolecules. 29 (1996) 6750-6760. doi:10.1021/ma951635z.

[18] Y. Tamai, H. Tanaka, K. Nakanishi, Molecular dynamics study of polymer-water interaction in hydrogels. 2. hydrogen-bond dynamics, Macromolecules. 29 (1996) 6761-6769. doi:10.1021/ma960961r.

[19] H. Du, R. Wickramasinghe, X. Qian, Effects of Salt on the Lower Critical Solution Temperature of Poly (N-Isopropylacrylamide), J. Phys. Chem. B. 114 (2010) 1659416604. doi:10.1021/jp105652c.

[20] H. Du, S.R. Wickramasinghe, X. Qian, Specificity in cationic interaction with poly(Nisopropylacrylamide), J. Phys. Chem. B. 117 (2013) 5090-5101. doi:10.1021/jp401817h.

[21] L.J. Abbott, A.K. Tucker, M.J. Stevens, Single chain structure of a poly(Nisopropylacrylamide) surfactant in water, J. Phys. Chem. B. 119 (2015) 3837-3845. doi:10.1021/jp511398q.

[22] A.K. Tucker, M.J. Stevens, Study of the polymer length dependence of the single chain transition temperature in syndiotactic Poly(N-isopropylacrylamide) oligomers in water, Macromolecules. 45 (2012) 6697-6703. doi:10.1021/ma300729z.

[23] S.A. Deshmukh, S.K.R.S. Sankaranarayanan, K. Suthar, D.C. Mancini, Role of solvation dynamics and local ordering of water in inducing conformational transitions in poly(N-isopropylacrylamide) oligomers through the LCST, J. Phys. Chem. B. 116 (2012) 2651-2663. doi:10.1021/jp210788u.

[24] J. Xu, Z. Zhu, S. Luo, C. Wu, S. Liu, First observation of two-stage collapsing kinetics of a single synthetic polymer chain, Phys. Rev. Lett. 96 (2006) 027802. doi:10.1103/PhysRevLett.96.027802.

[25] X. Ye, Y. Lu, L. Shen, Y. Ding, S. Liu, G. Zhang, C. Wu, How Many Stages in the Coil-to-Globule Transition of Linear Homopolymer Chains in a Dilute Solution?, Macromolecules. 40 (2007) 4750-4752. doi:10.1021/ma070167d.

[26] B. Hammouda, D. Jia, H. Cheng, Single-chain conformation for interacting Poly(Nisopropylacrylamide) in aqueous solution, Open Access J. Sci. Technol. 3 (2015). doi:10.11131/2015/101152.

[27] C. Wu, S. Zhou, Thermodynamically stable globule state of a single Poly(Nisopropylacrylamide) chain in water, Macromolecules. 28 (1995) 5388-5390. doi:10.1021/ma00119a036.

[28] C. Wu, A comparison between the 'Coil-to-Globule' transition of linear chains and the "volume phase transition" of spherical microgels, Polymer. 39 (1998) 4609-4619. doi:10.1016/S0032-3861(97)10130-6.

[29] G. Graziano, On the temperature-induced coil to globule transition of poly-Nisopropylacrylamide in dilute aqueous solutions, Int. J. Biol. Macromol. 27 (2000) 8997. doi:10.1016/S0141-8130(99)00122-1.

[30] W.L. Jorgensen, D.S. Maxwell, J. Tirado-Rives, Development and testing of the OPLS All-Atom force field on conformational energetics and properties of organic liquids, J. Am. Chem. Soc. 118 (1996) 11225-11236. doi:10.1021/ja9621760.

[31] H.J.C. Berendsen, J.R. Grigera, T.P. Straatsma, The missing term in effective pair potentials, J. Phys. Chem. 91 (1987) 6269-6271. doi:10.1021/j100308a038. 
[32] D. Van Der Spoel, E. Lindahl, B. Hess, G. Groenhof, A.E. Mark, H.J.C. Berendsen, GROMACS: fast, flexible, and free, J. Comput. Chem. 26 (2005) 1701-1718. doi:10.1002/jcc.20291.

[33] G.N. Patey, J.P. Valleau, A Monte Carlo method for obtaining the interionic potential of mean force in ionic solution, J. Chem. Phys. 63 (1975) 2334-2339. doi:10.1063/1.431685.

[34] G.M. Torrie, J.P. Valleau, Nonphysical sampling distributions in Monte Carlo freeenergy estimation: Umbrella sampling, J. Comput. Phys. 23 (1977) 187-199. doi:10.1016/0021-9991(77)90121-8.

[35] J. Kästner, Umbrella sampling, Wiley Interdiscip. Rev. Comput. Mol. Sci. 1 (2011) 932-942. doi:10.1002/wcms.66.

[36] S. Kumar, J.M. Rosenberg, D. Bouzida, R.H. Swendsen, P.A. Kollman, The weighted histogram analysis method for free-energy calculations on biomolecules. I. The method, J. Comput. Chem. 13 (1992) 1011-1021. doi:10.1002/jcc.540130812.

[37] J.S. Hub, B.L. de Groot, D. van der Spoel, g_wham-a free weighted histogram analysis implementation including robust error and autocorrelation estimates, J. Chem. Theory Comput. 6 (2010) 3713-3720. doi:10.1021/ct100494z.

[38] H.J.C. Berendsen, Simulating the Physical World: Hierarchical Modeling from Quantum Mechanics to Fluid Dynamics, Cambridge University Press, 2007.

[39] R. Freitag, F. Garret-Flaudy, Salt Effects on the Thermoprecipitation of Poly-(Nisopropylacrylamide) Oligomers from Aqueous Solution, Langmuir. 18 (2002) 34343440. doi:10.1021/la0106440.

[40] E.I. Tiktopulo, V.N. Uversky, V.B. Lushchik, S.I. Klenin, V.E. Bychkova, O.B. Ptitsyn, "Domain" Coil-Globule Transition in Homopolymers, Macromolecules. 28 (1995) 7519-7524. doi:10.1021/ma00126a032.

[41] S. Kunugi, T. Tada, N. Tanaka, K. Yamamoto, M. Akashi, Microcalorimetric Study of Aqueous Solution of a Thermoresponsive Polymer, poly(N-vinylisobutyramide) (PNVIBA), Polym. J. 34 (2002) 383-388. doi:10.1295/polymj.34.383.

[42] K. Otake, H. Inomata, M. Konno, S. Saito, Thermal analysis of the volume phase transition with N-isopropylacrylamide gels, Macromolecules. 23 (1990) 283-289. doi:10.1021/ma00203a049.

[43] T. Darden, D. York, L. Pedersen, Particle mesh Ewald: An N-log(N) method for Ewald sums in large systems, J. Chem. Phys. 98 (1993) 10089-10092. doi:10.1063/1.464397.

[44] G. Bussi, D. Donadio, M. Parrinello, Canonical sampling through velocity rescaling, J. Chem. Phys. 126 (2007) 014101. doi:10.1063/1.2408420.

[45] H.J.C. Berendsen, J.P.M. Postma, W.F. van Gunsteren, A. DiNola, J.R. Haak, Molecular dynamics with coupling to an external bath, J. Chem. Phys. 81 (1984) 3684-3690. doi:10.1063/1.448118.

[46] B. Roux, The calculation of the potential of mean force using computer simulations, Comput. Phys. Commun. 91 (1995) 275-282. doi:10.1016/0010-4655(95)00053-I. 
Abstract graphics

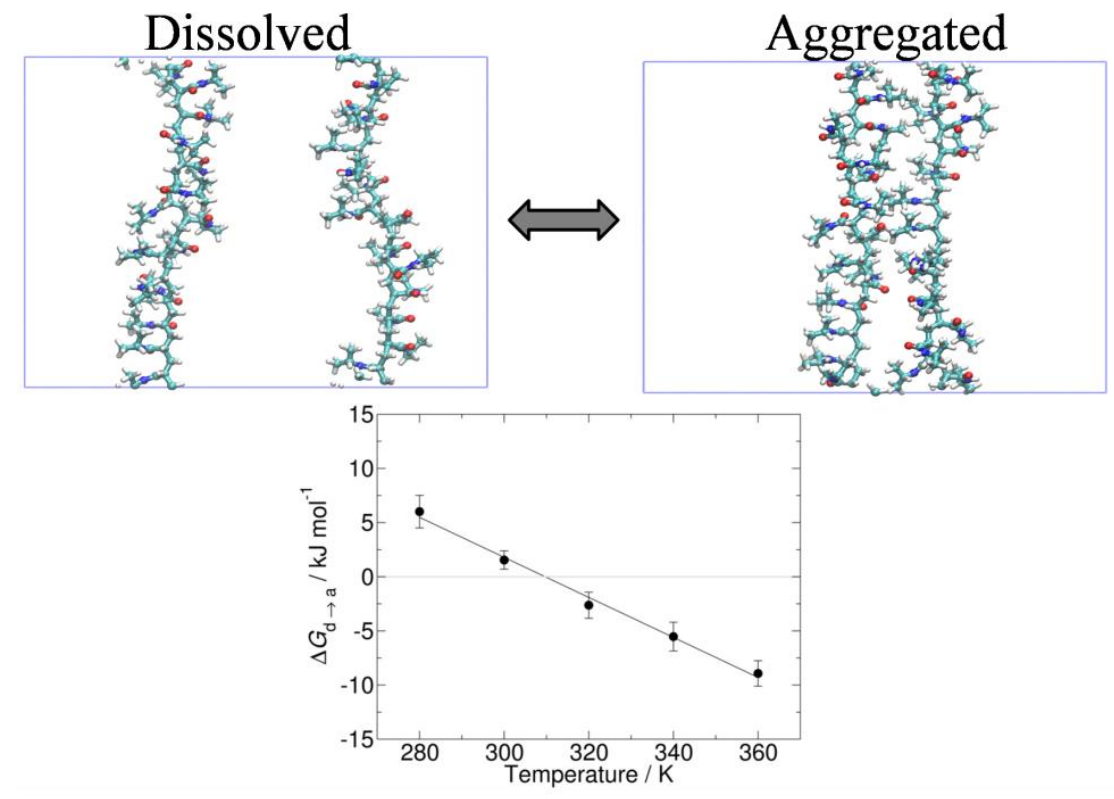

